\title{
Chancen und Perspektiven
}

\section{HERWIG BIRG}

\begin{abstract}
Deutschland nimmt eine Vorreiterrolle bei der Entwicklung hin zu einer schrumpfenden Bevölkerung ein. Seine Erfahrungen bei der notwendigen gesellschaftlichen und wirtschaftlichen Anpassung können für andere Länder wegweisend sein.
\end{abstract}

$\mathrm{D}_{\mathrm{s}}^{\mathrm{i}}$ ie demographischen Veränderungen in Deutschland sind seit langem absehbar und von der Wissenschaft seit über drei Jahrzehnten prognostiziert worden. Bei der Auseinandersetzung der Öffentlichkeit mit den demographisch bedingten Problemen gab es drei Phasen: In der langen Phase von der Mitte der siebziger Jahre des 20 . Jahrhunderts bis zu dessen Ende wurden die demographischen Probleme ignoriert, bestritten und tabuisiert. In der Folgephase, die jetzt zu Ende geht, wurden sie in ihrer Existenz zunehmend anerkannt und die Versäumnisse sowie die Verzögerungen zugegeben. In der dritten, zukünftigen Phase können Schlüsse für das Handeln gezogen werden.

Deutschland hat seit 1969 (neue Bundesländer) bzw. 1972 (alte Bundesländer) als erstes Land der Welt mehr Sterbefälle als Geburten. Es ist unter allen Staaten der Welt ein Vorreiter bei der Bevölkerungsschrumpfung (ohne Ausgleich durch Wanderungen) und gehört mit Japan zu den Ländern mit der intensivsten demographischen Alterung. Anders als Japan, das sich gegenüber Einwanderem verschließt, hat Deutschland im Vergleich mit den europäischen Nachbarländern die fünf- bis sechsfache Zahl an Zuwanderern aufgenommen. Trotz der teilweisen Kompensation des Geburtendefizits durch die Zuwanderung junger Menschen wirkt sich die demographische Alterung in Deutschland nachhaltiger und wesentlich früher aus als in allen vergleichbaren Nationen. Damit eröffnet sich die Möglichkeit, dass Deutschland im Umgang mit den demographisch bedingten Problemen Lösungen entwickelt, die später auch für andere Länder wichtig sein werden. Lassen sich aus der Schrumpfung und Alterung darüber hinaus noch andere konkrete Chancen ableiten?

Auf Gemeindeebene könnte eine solche darin bestehen, die nach dem Zweiten Weltkrieg vielerorts hastig wiedererrichteten Städte behutsam zu rekon- struieren und die Qualität ihrer architektonischen und gestalterischen Substanz wieder zu entdecken, um sie für künftige Generationen zu erhalten.

Die entscheidende Aufgabe liegt jedoch in einer erfolgreichen Integration der zugewanderten Bevölkerung - der künftigen Mehrheitsbevölkerung bei den Jüngeren in vielen Großstädten, sowie in einer Integration der Älteren in den Arbeitsmarkt und in neu zu entwickelnde soziale Dienste und Netzwerke.

Auf regionaler Ebene bietet sich die Gelegenheit, den jahrzehntelangen Trend zur Zersiedlung der Landschaft durch die Stadt-Umland-Wanderung („Suburbanisierung“) zu beenden und in eine „Reurbanisierung " umzukehren, um die Vitalität der Städte zu stärken. Dies bedeutet jedoch, dass gleichzeitig Siedlungen aufgelöst werden müssten, was Mut und planerische Weitsicht in einem bisher ungewohnten Maße voraussetzt.

Auf Landesebene ergibt sich die Chance, die vom Grundgesetz aufgegebene, seit Jahrzehnten aufgeschobene Neugliederung des Bundesgebiets in die Tat umzusetzen, um leistungsfähige Länder $z u$ schaffen. Die vom Grundgesetz geforderte Herstellung "gleichwertiger" (nicht gleichartiger) Lebensbedingungen in allen Teilen des Landes bietet die rechtliche Grundlage für die Verwirklichung schrumpfungsbedingter Reformen, vorausgesetzt, dass die Risiken nüchtern analysiert und nicht wie bisher ignoriert werden.

Auf nationaler Ebene und für jeden einzelnen liegt eine Chance der Schrumpfung und Alterung darin zu erkennen, wie stark wir von der solidarischen Unterstützung durch die nachrückenden, jüngeren Generationen abhängig sind, mit anderen Worten, dass es gute Gründe gibt, die Gesellschaft, in der wir leben, zu erhalten, indem wir ihre demographische Tragfähigkeit sichern. 


\section{Glossar: Demographische Grundbegriffe}

\section{Altenquotient:}

Zahl der Menschen im Alter von 60 und mehr Jahren auf 100 Menschen im Alter von 20 bis unter 60 . Statt der Altersschwellen 20/60 werden gelegentlich auch andere Schwellen wie $15 / 65$ verwendet.

Bestandserhaltungsniveau der Geburtenrate:

Diejenige Geburtenrate, die bei einem bestimmten Niveau der Mortalität langfristig die gleiche $Z$ ahl von Geburten und Sterbefällen zur Folge hat, so dass die Bevölkerungszahl (ohne Wanderungen) konstant ist.

Bevölkerungsbilanz:

Beispiel für eine Bevölkerungsbilanz: Bevölkerungszahl am 31. Dezember $2004=$ Bevölkerungszahl am 31. Dezember 2003 plus Zahl der Lebendgeborenen abzüglich Zahl der Sterbefälle plus Zahl der Zuwanderungen aus dem Ausland abzüglich Zahl der Abwanderungen ins Ausland (jeweils im Verlauf des Jahres 2004).

\section{Demographische Alterung:}

Anstieg des Durchschnittsalters einer Bevölkerung, gemessen durch den Altenquotienten oder das Medianalter.

Eigendynamik der Bevölkerungsentwicklung:

Hat eine Bevölkerung eine junge Altersstruktur, dann kann dies zu einem jahrzehntelangen (wenn auch nicht dauernden) Bevölkerungswachstum durch $\mathrm{Ge}$ burtenüberschüsse führen, obwohl die Fertilität bereits unter das Bestandserhaltungsniveau gefallen ist. Umgekehrt kann die Bevölkerung vorübergehend weiter schrumpfen, selbst wenn die Geburtenrate von einem niedrigen Wert aus bis über das Bestandserhaltungsniveau ansteigt. Der Einfluß der Altersstruktur auf Wachstum bzw. Schrumpfung der Bevölkerung wird auch mit den Begriffen "Schwung " und "Trägheit“. der Bevölkerung umschrieben.

\section{Fertilität:}

Fortpflanzungsverhalten einer Bevölkerung. Zur Messung der Fertilität dienen die Fertilitätsraten wie die Zusammengefasste Geburtenrate (siehe dort) und die jahrgangs- oder generationsspezifische Geburtenrate. Geburten:

Der Begriff „Geburten“ wird meist als Kürzel für „Zahl der Lebendgeborenen" verwendet. Infolge der Mehrlingsgeburten ist die Zahl der Lebendgeborenen größer als die Zahl der Geburtsvorgänge.

\section{Geburtenbilanz:}

Differenz zwischen der Zahl der Lebendgeborenen und der Zahl der Sterbefälle in einem bestimmten Zeitraum (meist Kalenderjahr). Ist die Differenz positiv (negativ), wird von Geburtenüberschuss (-defizit) gesprochen.

(rohe) Geburtenrate (-ziffer) und

altersspezifische Geburtenrate:

Im Deutschen wird der Quotient „Lebendgeborene auf 1000 Einwohner" als „rohe Geburtenrate“ oder als "rohe Geburtenziffer" bezeichnet. Wird diese nach den einzelnen Altersjahren des gebärfähigen Alters (15-45) untergliedert, ergeben sich die 31 „altersspezifischen Geburtenziffer" (Lebendgeborene pro Frau in einem bestimmten Alter auf 1000 Frauen dieses Alters). generatives Verhalten:

Gesamtheit aller ökonomischen, sozialen, kulturellen, psychologischen und rechtlichen Handlungs- und Ver- haltensbedingungen, einschließlich der Ziele und Wertvorstellungen der Menschen, von denen die Fertilität, gemessen durch die Zahl der Lebendgeborenen pro Frau/Mann, beeinflusst wird.

Jugendquotient:

Zahl der unter 20-Jährigen auf 100 Menschen im Alter von 20 bis unter 60 . Statt der Altersschwellen 20/60 werden gelegentlich auch andere Schwellen wie 15/65 verwendet.

Kohorte:

Meist bedeutungsgleich mit Geburtsjahrgang (Geburtsjahrgangskohorte). Daneben werden Heiratskohorten (Gesamtheit der Menschen, die im gleichen Jahr geheiratet haben) und andere Kohortenbegriffe unterschieden.

Lebenserwartung:

Durchschnittliche Lebensdauer in Jahren, dabei wird die "Lebenserwartung eines Neugeborenen" von der „ferneren Lebenserwartung“ für Menschen in einem bestimmten Alter unterschieden.

Medianalter:

Jede Bevölkerung lässt sich nach dem Alter in eine jüngere und eine ältere Hälfte teilen, das entsprechende Teilungsalter wird als "Medianalter" bezeichnet.

Migration:

Veränderung der Zahl und Zusammensetzung einer Bevölkerung eines bestimmten Gebietes durch $\mathrm{Zu}$ - und Abwanderungen gegenüber dem Ausland (Außenwanderungen) oder gegenüber anderen Regionen des gleichen Landes (Binnenwanderungen).

\section{Mortalität:}

Niveau der Sterblichkeit in einer Bevölkerung. Die Mortalität wird durch verschiedene demographische Kennziffern gemessen, zum Beispiel durch die alters- und geschlechtsspezifischen Sterberaten (bzw. -ziffern), das Medianalter und die Lebenserwartung.

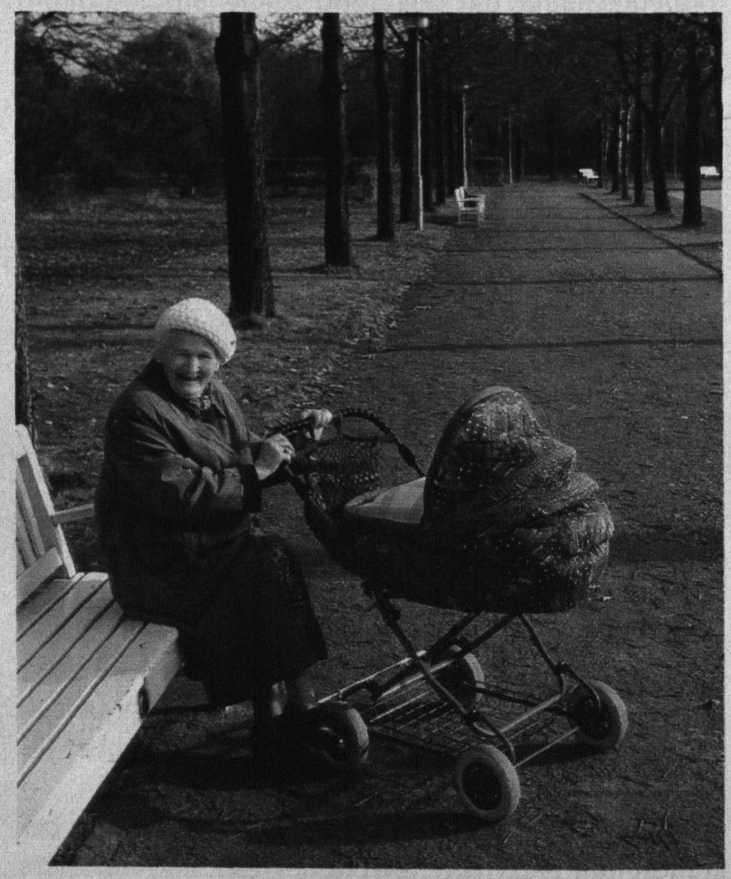

Foto: Sven Döring/VISUM 
Nettoreproduktionsrate (NRR):

Die Nettoreproduktionsrate misst, in welchem Maße eine Frauen- bzw. Elterngeneration durch ihre Nachkommen (ohne Wanderungen) ersetzt wird. Sie lässt sich messen durch die Zahl der Mädchen, die bei einem bestimmten Niveau der Mortalität pro Frau geboren werden. Ist die NRR größer als 1, wächst die Bevölkerung, ist sie kleiner als 1 , sinkt sie, bei einem Wert von 1 ist die Bevölkerung konstant. Bei der Bruttoreproduktionsrate (BRR) bleibt der Einfluss der Mortalität auf die Zahl der Geburten unberücksichtigt. Bei hoher Mortalität ist die Differenz zwischen der BRR und der NRR groß.

Sexualproportion:

Zahl der Einwohner männlichen Geschlechts auf 100 Einwohner weiblichen Geschlechts. Die Sexualproportion bei der Geburt beträgt rund 106 Jungen auf 100 Mädchen.

Unterstützungsquotient:

Summe aus Alten- und Jugendquotient.

(rohe) Sterbeziffer (-rate):

Die Sterberate oder -ziffer ist analog zur Geburtenrate (bzw. -ziffer) als Zahl der Sterbefälle auf 1000 Einwohner definiert (rohe Sterberate bzw. -ziffer). Bei Untergliederung des Zählers und Nenners der Sterberate nach Alter und Geschlecht ergeben sich die „altersund geschlechtsspezifischen Sterberaten (bzw. -ziffern)".

\section{Wachstumsrate der Bevölkerung:}

Veränderung der Bevölkerungszahl in einem Jahr in Prozent der Bevölkerungszahl am Anfang des Jahres. Bei konstanter Wachstumsrate ist das Bevölkerungswachstum "geometrisch" oder "exponentiell", bei steigender Wachstumsrate "hypergeometrisch“.

Wanderungsbilanz (oder -saldo):

Differenz zwischen der Zahl der Zuwanderungen und der Abwanderungen in einem bestimmten Zeitraum (meist Kalenderjahr).

Wanderungsrate:

Die Zuzugsrate (-ziffer) ist analog zur Geburtenrate als Zahl der Zuwanderungen auf 1000 Einwohner definiert. Entsprechend ist unter Fortzugsrate die Zahl der Abwanderungen auf 1000 Einwohner zu verstehen. Zusammengefasste Geburtenziffer (-rate)

Englisch: Total Fertility Rate (TFR) misst die Zahl der Lebendgeborenen je Frau oder je 1000 Frauen, wobei der Einfluss der Altersstruktur auf die Geburtenzahl ausgeschaltet wird, indem in jedem Alter von 15 bis 45 („gebärfähiges Alter") eine gleich große Gruppe von 1000 Frauen zugrunde gelegt wird. Die TFR lässt sich als Summe der altersspezifischen Geburtenziffern von 15 bis 45 berechnen. Sie gibt an, wieviel Geburten pro Frau entfallen, wenn eine gleich große Gruppe von je 1000 Frauen das gebärfähige Alter von 15 bis 45 in einem einzigen Kalenderjahr durchliefe und dabei die altersspezifischen Geburtenziffern dieses Jahres gelten würden.

\section{Literaturhinweise und Internetadressen}

Birg, Herwig: Die demographische Zeitenwende - Der Bevölkerungsrückgang in Deutschland und Europa, 3. Aufl., München 2003, $226 \mathrm{~S}$.

Einführung und Überblick über die Bevölkerungsentwicklung in Deutschland und Europa einschließlich der wichtigsten Auswirkungen auf die sozialen Sicherungssysteme, den Arbeitsmarkt, das Wirtschaftswachstum und andere Bereiche.

ders.: Die Weltbevölkerung - Dynamik und Gefahren, 2. Aufl., München 2004, $143 \mathrm{~S}$.

Gestraffter Überblick über die Geschichte der Weltbevölkerung einschließlich der Wechselwirkungen mit der Wissenschaftsgeschichte der Demographie.

Bundesinstitut für Bevölkerungsforschung (BiB): Bevölkerung. Fakten - Trends - Ursachen - Erwartungen. Die wichtigsten Fragen, Wiesbaden 2003.

Dieses Sonderheft der Schriftenreihe des Bundesinstituts für Bevölkerungsforschung bietet die wichtigsten Grunddaten und Analysen zur Bevölkerungsentwicklung Deutschlands.

Deutscher Bundestag, Referat Öffentlichkeitsarbeit ( $\mathrm{Hg}$.): Enquete-Kommission Demographischer Wandel - Herausforderungen unserer älter werdenden Gesellschaft für den Einzelnen und die Politik (Reihe "Zur Sache", Nr. 3), Berlin 2002, 691 S.

Der Bericht über die demographischen Grunddaten und die Vorausberechnungen bis 2050 behandelt das
Themenfeld der demographischen Auswirkungen mit den Schwerpunkten: Generationenverhältnis, Arbeit und Wirtschaft, Migration und Integration, Alterssicherung, Gesundheit, Pflege und soziale Dienste und bietet zu jedem dieser Bereiche Analysen als Grundlage für politische Handlungsstrategien.

Deutsche Stiftung Weltbevölkerung (Hg.): Weltbevölkerungsbericht 2003. Junge Menschen - Schlüssel zur Entwicklung, Stuttgart 2003, $92 \mathrm{~S}$.

Deutsche Ausgabe des jährlichen Weltbevölkerungsberichts des UN-Bevölkerungsfonds.

Hauser, Jürg A.: Bevölkerungs- und Umweltprobleme der Dritten Welt, Stuttgart Bd 11990,365 S., Bd 2 1991, 676 S. Informativer, breiter Überblick über die Auswirkungen der Bevölkerungsentwicklung auf die verschiedenen Bereiche der Umwelt, einschließlich von Konzeptionen für eine rationale Politik in den Industrie- und Entwicklungsländern.

Hessische Staatskanzlei (Hg.): Die Familienpolitik muss neue Wege gehen - ,Wiesbadener Entwurf' zur Familienpolitik, Wiésbaden $2003,528 \mathrm{~S}$.

Aufsatzsammlung zu einer familienpolitischen Strukturreform des Sozialstaates mit Stellungnahmen von Verbänden und Wissenschaftlern.

Höpflinger, François: Bevölkerungssoziologie - Eine Einführung in bevölkerungssoziologische Ansätze und demo- 
graphische Prozesse, Weinheim und München 1997, $232 \mathrm{~S}$. Das Buch enthält fundierte Analysen des demographischen und gesellschaftlichen Wandels und stellt die wesentlichen theoretischen Erklärungsansätze des generativen Verhaltens, der Migration, der Mortalität und der Lebenserwartung dar.

Leipert, Christian ( $\mathrm{Hg}$.$) : Demographie und Wohlstand -$ Neuer Stellenwert für Familie in Wirtschaft und Gesellschaft, Opladen 2003, $304 \mathrm{~S}$.

Grundlegender Sammelband mit Aufsätzen über die demographischen Wirkungen auf Wirtschaft und Gesellschaft und die Reformvorschläge auf den Gebieten der-Familien-, Sozial- und Wirtschaftspolitik.

Malthus, Thomas Robert: An Essay on the Principle of Population, London 1798 und 1803 (erweiterte Ausgabe). Deutsche Übersetzung von Chr. M. Barth: „Das Bevölkerungsgesetz“, München 1977.

Grundlegendes Werk der klassischen Periode der Bevölkerungstheorie. Die zweite, wesentlich erweiterte Ausgabe von 1803 enthält eine Fülle von statistischen Belegen und eine gründliche und detaillierte Darlegung der zentralen Thesen, die bis heute ihre Wirkung entfalten.

Schmid, Josef/Heigl, Andreas/Mai, Ralf: Sozialprognose Die Belastung der nachwachsenden Generationen, München 2000, $213 \mathrm{~S}$.

Guter Einstieg und informativer Überblick über die Auswirkungen der demographischen Alterung und Bevölkerungsschrumpfung auf Staat, Wirtschaft und soziale Sicherungssysteme in Deutschland.

Süßmilch, Johann Peter: Die göttlliche Ordnung in den Veränderungen des menschlichen Geschlechts, aus der $\mathrm{Ge}$ burt, Tod und Fortpflanzung desselben erwiesen, Berlin 1741 und 1762 (erweiterte Ausgabe)

Hauptwerk der klassischen Bevölkerungstheorie. Darin wird die Frage nach der "Tragfähigkeit der Erde" zum ersten Mal gestellt und auf eine in Methodik und wissenschaftlicher Herangehensweise beinahe moderne Art und Weise beantwortet.
Wingen, Max: Familienpolitik - Grundlagen und aktuelle Probleme, Stuttgart 1997, $485 \mathrm{~S}$.

Dieses Buch bietet Informationen und Anregungen für eine rationale Familienpolitik, es beschreibt international vergleichend die Ziele und die wichtigsten $\mathrm{Maß}$ nahmenbereiche, Träger und Wirkungen der Familienpolitik.

United Nations, Population Division (Hg.): Replacement Migration - Is it a solution to declining and aging populations? New York 2001, $151 \mathrm{~S}$.

Grundlegendes Werk mit demographischen Analysen und Projektionsrechnungen für acht Länder (einschließlich Deutschland) sowie für Europa und die Europäische Union, wobei die Frage im Zentrum steht, welche demographischen Folgen sich ergeben, wenn die durch Tod ausscheidenden Generationen nicht durch Geburten im Inland erneuert, sondern durch Einwanderungen aus dem Ausland ersetzt (,replacement") werden.

\section{Internetadressen:}

www.bib-demographie.de

Bundesinstitut für Bevölkerungsforschung beim Statistischen Bundesamt.

www.destatis.de

Statistisches Bundesamt Wiesbaden. Bevölkerung Deutschlands bis 2050

10. Koordinierte Bevölkerungsberechnung.

www.dsw-online.de

Deutsche Stiftung Weltbevölkerung, Hannover.

www.ibs.uni-bielefeld.de

Institut für Bevölkerungsforschung und Sozialpolitik. Bevölkerungsprognosen und Simulationsrechnungen zur Bevölkerungsentwicklung in Deutschland.

www.un.org/esa/population/unpop.htm Website der Bevölkerungsabteilung der UN. 